\title{
Effects of Background Risks on Cautiousness with an Application to a Portfolio Choice Problem
}

\author{
Chiaki Hara ${ }^{1}$ \\ Institute of Economic Research, Kyoto University \\ James Huang ${ }^{2}$ \\ Department of Accounting and Management \\ Lancaster University Management School \\ Christoph Kuzmics ${ }^{3,4}$ \\ MEDS, Kellogg School of Management, Northwestern University
}

March 31, 2008

\footnotetext{
${ }^{1}$ The corresponding author. Address: Yoshida-Honmachi, Sakyo-ku, Kyoto 606-8501 Japan. Fax: +81 (0)75 753 7148. Email: hara@kier.kyoto-u.ac.jp.

${ }^{2}$ The email address is james.huang@lancaster.ac.uk.

${ }^{3}$ The email address is c-kuzmics@kellogg.northwestern.edu.

${ }^{4}$ We are also grateful to Jeremy Edwards, Günter Franke, Christian Gollier, Toshiki Honda, Atsushi Kajii, Takashi Kamihigashi, Peter Klibanoff, Kazuhiko Ohashi, Heracles Polemarchakis, Makoto Saito, Karl Schmedders, and Jan Werner; and seminar/conference participants at Berkeley, Cambridge, Hitotsubashi, Keio, Kobe, Kumamoto, Kyoto, London, Oita, Osaka, Rhodes Island, Toyama, Vigo, and Waseda. Hara gratefully acknowledges the financial assistance from the following funding bodies: Grand in Aid for Scientific Research (B) from Japan Society for the Promotion of Sciences on "Development of the Collective Risk Management in Large Scale Portfolio"; Grant in Aid for Specially Promoted Research from Japan Society for the Promotion of Sciences for "Economic Analysis on Intergenerational Problems"; Inamori Foundation on "Efficient Risk-Sharing: An Application of Finance Theory to Development Economics"; and Murata Science Foundation on "Internationalization of Asset Markets and Investors' Portfolio Choice Behavior".
} 


\begin{abstract}
We provide a necessary and a sufficient condition on an individual's expected utility function under which any zero-mean idiosyncratic risk increases cautiousness (the derivative of the reciprocal of the absolute risk aversion), which is the key determinant for this individual's demand for options and portfolio insurance.
\end{abstract}

JEL Classification Codes: D51, D58, D81, G11, G12, G13.

Keywords: Risk aversion, risk tolerance, cautiousness, portfolio insurance, idiosyncratic risks, background risks, incomplete markets. 


\section{Introduction}

Gollier and Pratt (1996) termed an expected utility function risk-vulnerable if any zeromean background risk increases its Arrow-Pratt measure of absolute risk aversion at every consumption level. ${ }^{1}$ In other words, any gamble the decision maker turns down in the absence of any background risk she will also turn down in the presence of a zeromean background risk. This has the consequence that the decision maker's demand for the risky asset decreases in the presence of a zero-mean background risk in the standard portfolio choice problem where there only two assets to choose from, one risk-free (bonds) and one risky (equity).

The decision maker would often have a much larger set of assets to choose from. In a more general portfolio choice problem in which there are, in addition, derivative assets, such as options, written on the risky asset, the decision maker's consumption resulting from her optimal portfolio is typically a non-affine function of the payoff of the risky asset. This is the case when the decision maker's portfolio includes not only bonds and equity but also options. Conversely, once we know her optimal consumption as a function of the payoff of the risky asset, we can work out how she can implement this plan by holding a portfolio of the bond, equity, and a set of call options of various strike prices. If the optimal consumption is a non-affine function of the payoff of the risky asset then this portfolio must include options. Moreover, it can be shown that the more curvature this function has the higher the decision maker's wealth share invested in options.

While the curvature of the decision maker's optimal consumption function determines her demand for options, this curvature is, in turn, determined not so much by her degree of risk aversion but rather of cautiousness, the derivative of the absolute risk tolerance, which in turn is the reciprocal of the Arrow-Pratt measure of absolute risk aversion. This was shown in Chevallier and Müller (1994) and also Hara, Huang, and Kuzmics (2007). The more cautious the decision maker is the more convex (relative to the slope) is her optimal consumption function. Hence, the more cautious she is the more she demands options relative to the risky asset.

The question we are addressing in this paper then is under what conditions on the decision maker's utility function she demands more options (portfolio insurance) if she

\footnotetext{
${ }^{1}$ To be exact, they termed an expected utility function risk-vulnerable if any background risk having non-positive mean increases its Arrow-Pratt measure of absolute risk aversion at every consumption level.
} 
faces any independent zero-mean background risk, i.e., under what conditions on her utility function a non-hedgeable background risk makes her more cautious. This question is very much analogous to Gollier and Pratt (1996)'s question, alluded to above, of what makes a utility function risk-vulnerable.

The main results of this paper are Theorems 1, 2, and 3 . Theorem 1 provides three equivalent essentially necessary and sufficient conditions for cautiousness to be increased under small background risks. The first is simply in terms of higher order derivatives of the utility function. The second is in terms of the decision maker's risk tolerance and its higher-order derivatives, while the third is purely in terms of the decision maker's absolute risk aversion and its higher-order derivatives. All these equivalent conditions are somewhat complicated. Under the additional assumption that the decision maker is prudent (i.e. her utility function has a positive third derivative), which is often assumed, Theorem 2 provides more insightful equivalent conditions for increased cautiousness under small risks: a utility function exhibits increased cautiousness under small risks if and only if the negative of its first derivative exhibits a higher degree of risk-vulnerability, in the sense of equation (10) of Gollier and Pratt (1996), than the original utility function itself. Finally, Theorem 3 provides a global sufficient condition in terms of cautiousness and its derivatives. In fact a sufficient condition for increased cautiousness under any zero-mean background risk is that cautiousness be non-negative, non-increasing and convex. The first condition is nothing but decreasing absolute risk aversion (DARA), while the second implies convex risk aversion, which together with the first implies the sufficient condition for risk-vulnerability (Corollary 1 of Gollier and Pratt (1996)). Especially given the third condition the sufficient condition for increased cautiousness under any background risk provided in Theorem 3 is stronger than that for risk-vulnerability. Finally to make sure that our results are not vacuous we should add that any utility function exhibiting hyperbolic absolute risk aversion (HARA) satisfies our sufficient condition.

The observation that curvature of the consumption function is related to the demand for options ${ }^{2}$ was first made by Leland (1980) and Brennan and Solanki (1981). Section 6, following Leland (1980)'s approach, establishes that the second derivative of the consumption function can be interpreted as the density function of strike prices of

\footnotetext{
${ }^{2}$ Huang and Stapleton (2007) similarly established that cautiousness is the key determinant for the demand for options even in the simpler portfolio choice problem in which the decision maker has the choice between three assets only, the risk-free asset, a risky asset, and a single option with a fixed strike price.
} 
options that the decision maker holds to implement her consumption function, as long as the first derivative function is of bounded variation. Section 6 then provides the motivating result for the main part of this paper that an increase in the decision maker's cautiousness increases her demand for options relative to her demand for the risky asset.

Franke et al. (1998) were the first to point out the possibility that background risk might increase a consumer's cautiousness, and, hence, her demand for options. They established this result for the case when the decision maker has a utility function from the HARA class for which cautiousness is constant. We extend their result in this paper by providing general conditions on utility functions that either are necessary or sufficient for this result to be true.

The main part of the paper then proceeds as follows. Section 2 formulates our problem. Section 3 shows that cautiousness is increased under any zero-mean background risk if and only if it is increased under any two-point zero-mean background risk. This is similar to Lemma 1 in Gollier (2001a) and related to the diffidence theorem of Gollier and Kimball (1996), see also section 6.1 in Gollier (2001a). Section 4 provides a necessary and sufficient condition on the utility function to exhibit increased cautiousness under any small zero-mean risk. In this section we also provide a brief discussion of what it exactly means for a risk to be small. This condition obviously also serves as a necessary condition for increased cautiousness under all zero-mean background risks, small or not. Section 5 finally provides a sufficient condition on the utility function that ensures that cautiousness is increased under all zero-mean background risks, small or not.

\section{Setup}

Let $v:(\underline{c}, \infty) \rightarrow \boldsymbol{R}$ be a von-Neumann Morgenstern utility function (also known as Bernoulli utility function), where $\underline{c}>-\infty$. Note that the domain is assumed to be bounded from below but not from above. Let $v$ be at least twice continuously differentiable ${ }^{3}$ and satisfy $v^{\prime}(x)>0$ and $v^{\prime \prime}(x)<0$ for every $x>\underline{c}$, and let $v$ satisfy the Inada conditions, $v^{\prime}(x) \rightarrow \infty$ as $x \rightarrow \underline{c}$ and $v^{\prime}(x) \rightarrow 0$ as $x \rightarrow \infty$.

We assume that there is a consumer with this utility function $v$, who in addition to being exposed to tradeable macroeconomic risk (which we do not explicitly model), also faces an independent background risk $\xi$, which is assumed to be stochastically

\footnotetext{
${ }^{3}$ The degree of continuous differentiability necessary for each of the subsequent results will be made clear in its proof.
} 
independent of the market risk, and which the consumer cannot trade, i.e. is forced to absorb. This risk is described by a probability measure space $(\Theta, \mathscr{G}, Q)$, for which the expectation operator is denoted by $E^{Q}$ or just $E$. The cumulative distribution function of $\xi$ is denoted by $G: \boldsymbol{R} \rightarrow[0,1]$. For simplicity, we use the following assumptions throughout the paper. First, the support of the distribution of $G$ is bounded, that is, there are two numbers $\underline{e}$ and $\bar{e}$ such that $G(\underline{e})=0$ and $G(\bar{e})=1$. Second, $\xi$ has zero mean, that is, $\int_{e}^{\bar{e}} y d G(y)=0$. The first assumption guarantees that all the expected values that we consider in the subsequent analysis are well defined and Leibnitz's rule is applicable, so that the order of integration and differentiation for smooth functions can be swapped. The second is a normalization and implies that $\underline{e} \leq 0$ and $\bar{e} \geq 0$.

Following Kihlstrom et al. (1981) and Nachman (1982), we can define the consumer's induced utility function

$$
u(x)=E(v(x+\xi)) .
$$

This is the utility function the consumer takes to the market, i.e. he makes decisions about what assets to buy on the basis of the induced utility function.

In this reformulation, the realized consumption level, inclusive of the realized background risk, must of course be in the domain $(\underline{c}, \infty)$ almost surely. To guarantee this, we concentrate on the consumption levels $x>\underline{c}-\underline{e}$. Denote $\underline{d}=\underline{c}-\underline{e}$, then the domain of the induced utility function $u$ is $\left[\underline{d}_{0}, \infty\right)$.

Define the consumer's original (absolute) risk tolerance, $s:(\underline{c}, \infty) \rightarrow \boldsymbol{R}_{++}$, by

$$
s(x)=-\frac{v^{\prime}(x)}{v^{\prime \prime}(x)} .
$$

This is just the reciprocal of the consumer's Arrow-Pratt coefficient of absolute risk aversion $a(x)=-v^{\prime \prime}(x) / v^{\prime}(x)$. The (absolute) risk tolerance of the corresponding induced utility function $u$ shall be denoted by $t:(\underline{c}, \infty) \rightarrow \boldsymbol{R}_{++}$. By Leibnitz's rule,

$$
t(x)=-\frac{E\left(v^{\prime}(x+\xi)\right)}{E\left(v^{\prime \prime}(x+\xi)\right)} .
$$

Following the terminology coined by Wilson (1968) the derivative of risk tolerance shall be called (absolute) cautiousness. The consumer's original cautiousness is therefore given by $s^{\prime}(x)$, while the consumer's induced (absolute) cautiousness is given by $t^{\prime}(x)$ for $x$ in the respective domain. 
Denote by $\psi(x)$ the prudence of $v$ of Kimball (1990):

$$
\psi(x)=-\frac{v^{\prime \prime \prime}(x)}{v^{\prime \prime}(x)}
$$

Also denote by $\varphi$ the prudence of the induced utility function $u$, then we have

$$
\varphi(x)=-\frac{E\left(v^{\prime \prime \prime}(x+\xi)\right)}{E\left(v^{\prime \prime}(x+\xi)\right)} .
$$

The following relationship among the risk tolerance, prudence, and cautiousness is easy to prove and yet useful.

Lemma 1 1. For every $x>\underline{c}, s^{\prime}(x)=s(x) \psi(x)-1$.

2. For every $x>\underline{d}, t^{\prime}(x)=t(x) \varphi(x)-1$.

Gollier and Pratt (1996, Propositions 2 and 3) gave sufficient conditions under which, if $\xi$ has a positive variance, then $t(x) \leq s(x)$, that is, the background risk makes the consumer less risk tolerant (more risk averse). They called utility functions having this property risk vulnerable. The aim of this paper is to provide conditions on risk-tolerance and its derivatives for $t^{\prime}(x) \geq s^{\prime}(x)$ for any zero-mean background risk.

\section{Two-point background risks}

In this section we attempt to provide necessary and sufficient conditions for the cautiousness to increase under any zero-mean background risk, that is,

$$
\frac{E\left(v^{\prime}(x+\xi)\right) E\left(v^{\prime \prime \prime}(x+\xi)\right)}{\left(E\left(v^{\prime \prime}(x+\xi)\right)\right)^{2}} \geq \frac{v^{\prime}(x) v^{\prime \prime \prime}(x)}{\left(v^{\prime \prime}(x)\right)^{2}}
$$

or equivalently,

$$
\left(v^{\prime \prime}(x)\right)^{2} E\left(v^{\prime}(x+\xi)\right) E\left(v^{\prime \prime \prime}(x+\xi)\right)-v^{\prime}(x) v^{\prime \prime \prime}(x)\left(E\left(v^{\prime \prime}(x+\xi)\right)\right)^{2} \geq 0 .
$$

As for risk-vulnerability, (Gollier and Pratt, 1996, Proposition 2) gave an equivalent condition, involving the absolute risk aversion, its derivative, and marginal utilities, which are evaluated at two distinct consumption levels. Unfortunately, we do not know whether there is any such equivalent condition for the cautiousness to be increased by 
any zero-mean background risk, involving the higher-order derivatives of $s$, evaluated at two distinct consumption levels.

The nature of the difficulty is as follows: (Gollier and Pratt, 1996, Proposition 2) is an application of the diffidence theorem, (Gollier, 2001a, Proposition 11 of Section 6), which, in turn, is based on the separating hyperplane theorem. As is well known, for the latter theorem to be applicable, the relevant sets or functions need to be convex; and this prerequisite is met for the inequality defining risk vulnerability $t(x) \leq s(x)$, or, equivalently,

$$
v^{\prime \prime}(x) E\left(v^{\prime}(x+\xi)\right)-v^{\prime}(x) E\left(v^{\prime \prime}(x+\xi)\right) \geq 0,
$$

because the left-hand side is linear in expectations. The left-hand side of our inequality (2), in contrast, is quadratic in expectations, and the diffidence theorem cannot immediately be applied to obtain an equivalent condition for increased cautiousness.

Although the diffidence theorem is not immediately applicable, the fact that the left-hand side of (2) is quadratic allows us to derive the following equivalent condition (Proposition 1) for increased cautiousness by two-point zero-mean background risks. Define $Z$ as the set of all mean-zero background risks of bounded support, and $Z^{\mathrm{b}}$ as the set of all $\xi \in Z$ of which the supports of the distributions consist of at most two points.

Proposition 1 Suppose that $v^{\prime \prime \prime}(x) \geq 0$ for every $x>\underline{c}$. Then the cautiousness is increased, i.e. $t^{\prime}(x) \geq s^{\prime}(x)$ for every $x>\underline{c}$, by every $\xi \in Z$ if and only if it is increased by every $\xi \in Z^{\mathrm{b}}$.

The cautiousness is increased by every background risk if and only if the infimum of the left-hand side of (2) with respect to all $\xi \in Z$ is non-negative. The proof of this proposition boils down to showing that although the set $Z^{\mathrm{b}}$ is strictly smaller than $Z$, the infimum of the left-hand side of (2) with respect to all $\xi \in Z^{\mathrm{b}}$ is equal to the infimum of the left-hand side of (2) with respect to all $\xi \in Z$. This sort of property is well known for the minimization problem of which the objective function is linear in expectations, such as the left-hand side of (3). ${ }^{4}$ The crux of the proof lies in proving that the same property holds for a quadratic function, such as the left-hand side of (2).

Proof of Proposition 1 It suffices to show that if inequality (1) is satisfied for every $\xi \in Z^{\mathrm{b}}$, then it is satisfied for every $\xi \in Z$. This will be done in three stages. In the first

\footnotetext{
${ }^{4}$ This is stated, for example, as (Gollier, 2001a, Lemma 1).
} 
step we will prove that if inequality (1) is satisfied for every $\xi \in Z^{\mathrm{b}}$, then

$$
\begin{aligned}
& E\left[v^{\prime}\left(x+\xi_{1}\right)\right] E\left[v^{\prime \prime \prime}\left(x+\xi_{2}\right)\right]+E\left[v^{\prime}\left(x+\xi_{2}\right)\right] E\left[v^{\prime \prime \prime}\left(x+\xi_{1}\right)\right] \\
& -2 \frac{v^{\prime}(x) v^{\prime \prime \prime}(x)}{\left(v^{\prime \prime}(x)\right)^{2}} E\left[v^{\prime \prime}\left(x+\xi_{1}\right)\right] E\left[v^{\prime \prime}\left(x+\xi_{2}\right)\right] \geq 0
\end{aligned}
$$

for every pair $\left(\xi_{1}, \xi_{2}\right) \in Z^{\mathrm{b}} \times Z^{\mathrm{b}}$. We then show in the second step that if inequality (4) is satisfied for every pair $\left(\xi_{1}, \xi_{2}\right) \in Z^{\mathrm{b}} \times Z^{\mathrm{b}}$, then it must be satisfied for every pair $\left(\xi_{1}, \xi_{2}\right) \in Z \times Z$. The final step is then to show that if inequality (4) is satisfied for every pair $\left(\xi_{1}, \xi_{2}\right) \in Z \times Z$, then inequality (1) is satisfied for every $\xi \in Z$.

Step 1: Since the arithmetic mean is no less than the geometric mean,

$$
\begin{aligned}
& \frac{E\left[v^{\prime}\left(x+\xi_{1}\right)\right] E\left[v^{\prime \prime \prime}\left(x+\xi_{2}\right)\right]+E\left[v^{\prime}\left(x+\xi_{2}\right)\right] E\left[v^{\prime \prime \prime}\left(x+\xi_{1}\right)\right]}{E\left[v^{\prime \prime}\left(x+\xi_{1}\right)\right] E\left[v^{\prime \prime}\left(x+\xi_{2}\right)\right]} \\
& \geq 2 \sqrt{\frac{E\left[v^{\prime}\left(x+\xi_{1}\right)\right] E\left[v^{\prime \prime \prime}\left(x+\xi_{2}\right)\right]}{E\left[v^{\prime \prime}\left(x+\xi_{1}\right)\right] E\left[v^{\prime \prime}\left(x+\xi_{2}\right)\right]} \frac{E\left[v^{\prime}\left(x+\xi_{2}\right)\right] E\left[v^{\prime \prime \prime}\left(x+\xi_{1}\right)\right]}{E\left[v^{\prime \prime}\left(x+\xi_{1}\right)\right] E\left[v^{\prime \prime}\left(x+\xi_{2}\right)\right]}} \\
& =2 \sqrt{\frac{E\left[v^{\prime}\left(x+\xi_{1}\right)\right] E\left[v^{\prime \prime \prime}\left(x+\xi_{2}\right)\right]}{\left[E\left[v^{\prime \prime}\left(x+\xi_{1}\right)\right]\right]^{2}} \frac{E\left[v^{\prime}\left(x+\xi_{2}\right)\right] E\left[v^{\prime \prime \prime}\left(x+\xi_{1}\right)\right]}{\left[E\left[v^{\prime \prime}\left(x+\xi_{2}\right)\right]\right]^{2}}} .
\end{aligned}
$$

for every pair $\left(\xi_{1}, \xi_{2}\right) \in Z^{\mathrm{b}} \times Z^{\mathrm{b}}$. By applying inequality (1) twice (for $\xi_{1} \in Z^{\mathrm{b}}$ and $\xi_{2} \in Z^{\mathrm{b}}$ ), we see that the far right-hand side is no less than

$$
2 \sqrt{\left(\frac{v^{\prime}(x) v^{\prime \prime \prime}(x)}{\left(v^{\prime \prime}(x)\right)^{2}}\right)^{2}}=2 \frac{v^{\prime}(x) v^{\prime \prime \prime}(x)}{\left(v^{\prime \prime}(x)\right)^{2}} .
$$

Thus inequality (4) holds for every pair $\left(\xi_{1}, \xi_{2}\right) \in Z^{\mathrm{b}} \times Z^{\mathrm{b}}$.

Step 2: It suffices to show that for every pair $\left(\xi_{1}^{*}, \xi_{2}^{*}\right) \in Z \times Z$, there exists a pair $\left(\xi_{1}, \xi_{2}\right) \in$ $Z^{\mathrm{b}} \times Z^{\mathrm{b}}$ such that the two pairs give rise to the same value of the left-hand side of (4). So let $\left(\xi_{1}^{*}, \xi_{2}^{*}\right) \in Z \times Z$ and consider the function

$$
\begin{gathered}
\xi_{2} \mapsto E\left[v^{\prime}\left(x+\xi_{1}^{*}\right)\right] E\left[v^{\prime \prime \prime}\left(x+\xi_{2}\right)\right]+E\left[v^{\prime}\left(x+\xi_{2}\right)\right] E\left[v^{\prime \prime \prime}\left(x+\xi_{1}^{*}\right)\right] \\
-2 \frac{v^{\prime}(x) v^{\prime \prime \prime}(x)}{\left(v^{\prime \prime}(x)\right)^{2}} E\left[v^{\prime \prime}\left(x+\xi_{1}^{*}\right)\right] E\left[v^{\prime \prime}\left(x+\xi_{2}\right)\right]
\end{gathered}
$$

of $\xi_{2}$. This is a linear function in the expectations for $\xi_{2}$. Thus there exists a 
$\xi_{2} \in Z^{\mathrm{b}}$ such that $\left(\xi_{1}^{*}, \xi_{2}\right)$ and $\left(\xi_{1}^{*}, \xi_{2}^{*}\right)$ give rise to the same value of the left-hand side of (4). Then, by applying the same argument with respect to $\xi_{1}$, we can show that there exists a $\xi_{1} \in Z^{\mathrm{b}}$ such that $\left(\xi_{1}, \xi_{2}\right)$ and $\left(\xi_{1}^{*}, \xi_{2}\right)$ give rise to the same value of the left-hand side of $(4)$. Thus $\left(\xi_{1}, \xi_{2}\right)$ and $\left(\xi_{1}^{*}, \xi_{2}^{*}\right)$ give rise to the same value of the left-hand side of (4).

Step 3: Inequality (1) follows from inequality (4) taking $\xi_{1}=\xi_{2}=\xi \in Z$ in inequality (4).

\section{Necessary and sufficient conditions for small risks}

Gollier and Pratt (1996, Equation (12)) provide necessary and sufficient conditions for risk aversion to increase under any small zero-mean background risk. Their proof is based on Taylor series expansions on relevant derivatives of the utility function $v$. We can similarly provide an analogous result of this necessary and sufficient condition for small background risks for the question at hand. The utility function $v$ is real analytic if for each point in the domain of $v$, there exists a neighborhood of the point on which $v$ coincides with an absolutely convergent power series. Every real analytic utility function is infinitely many times differentiable but the converse does not hold. Yet the class of real analytic utility functions is broad enough to contain all utility functions exhibiting hyperbolic absolute risk aversion.

Theorem 1 Let $\left(\xi_{m}\right)_{m}$ be a sequence of random variables with mean zero and support in the interval $\left[-b_{m}, b_{m}\right]$ for every $m$, where $b_{m} \rightarrow 0$ as $m \rightarrow \infty$. Assume that $v$ is real analytic and denote by $t_{m}$ by the induced risk tolerance of the decision maker having the background risk $\xi_{m}$. Then, for every $x>\underline{c}$, if $t_{m}^{\prime}(x) \geq s^{\prime}(x)$ for infinitely many $m$, then

$$
v^{\prime \prime}(x)\left(v^{\prime \prime \prime}(x)\right)^{2}+v^{\prime}(x) v^{\prime \prime}(x) v^{(5)}(x)-2 v^{\prime}(x) v^{\prime \prime \prime}(x) v^{(4)}(x) \leq 0,
$$

or, equivalently,

$$
(s(x))^{2} s^{\prime \prime \prime}(x)-2 s(x)\left(1+2 s^{\prime}(x)\right) s^{\prime \prime}(x)+2\left(s^{\prime}(x)\right)^{2}\left(1+s^{\prime}(x)\right) \leq 0,
$$


or, equivalently,

$$
a(x) a^{\prime \prime \prime}(x)-2\left(a^{\prime}(x)+(a(x))^{2}\right) a^{\prime \prime}(x)+2 a(x)\left(a^{\prime}(x)\right)^{2} \leq 0 .
$$

Conversely if either (and, hence, all) of the above three inequalities holds as a strict inequality, then $t_{m}^{\prime}(x)>s^{\prime}(x)$ for every sufficiently large $m$.

Proof of Theorem 1 Let $x \in(\underline{c}, \infty)$ and $b \in(0, x-\underline{c})$ be such that $v$ is an absolutely convergent power series on $(x-b, x+b)$. Then, for every $n=1,2, \ldots, v^{(n)}$ is also an absolutely convergent power series on $(x-b, x+b)$ and, more specifically, can be written as

$$
v^{(n)}(x+z)=\sum_{k=0}^{\infty} \frac{v^{(n+k)}(x)}{k !} z^{k}
$$

for every $z \in(-b, b)$.

We can assume without loss of generality that $E\left(\xi_{m}^{2}\right)>0$ and $0<b_{m}<\min \{b, 1\}$ for every $m$. Then $\left|\xi_{m}\right|^{k} \leq b_{m}\left|\xi_{m}\right|^{k-1}$ and hence $E\left(\left|\xi_{m}\right|^{k}\right) \leq b_{m} E\left(\left|\xi_{m}\right|^{k-1}\right)$. Thus $\left|E\left(\xi_{m}^{k}\right)\right| \leq b_{m}^{k}<b^{k}$ for every $k \geq 1$ and the infinite series

$$
\sum_{k=0}^{\infty} \frac{v^{(n+k)}(x)}{k !} E\left(\xi_{m}^{k}\right)
$$

is absolutely convergent. Moreover, as $m \rightarrow \infty$,

$$
E\left(\xi_{m}^{k}\right) \rightarrow 0 \text { for every } k \geq 2 \text { and } \frac{E\left(\xi_{m}^{k}\right)}{E\left(\xi_{m}^{2}\right)} \rightarrow 0 \text { for every } k \geq 3 .
$$

Write

$$
\sigma_{m}^{2}=E\left(\xi_{m}^{2}\right) \text { and } \gamma_{m}^{n}=\frac{1}{\sigma_{m}^{2}} \sum_{k=3}^{\infty} \frac{v^{(n+k)}(x)}{k !} E\left(\xi_{m}^{k}\right) .
$$

By (8), as $m \rightarrow \infty, \sigma_{m}^{2} \rightarrow 0$ and $\gamma_{m}^{n} \rightarrow 0$ for every $n$. By the dominated convergence theorem,

$$
E\left(v^{(n)}\left(x+\xi_{m}\right)\right)=\sum_{k=0}^{\infty} \frac{v^{(n+k)}(x)}{k !} E\left(\xi_{m}^{k}\right)=v^{(n)}(x)+\left(v^{(n+2)}(x)+\gamma_{m}^{n}\right) \sigma_{m}^{2}
$$

for every $n$ and every $m$.

Suppose that $t_{m}^{\prime}(x) \geq s^{\prime}(x)$ for infinitely many $m$. By taking a subsequence if 
necessary, we can assume that $t_{m}^{\prime}(x) \geq s^{\prime}(x)$ for every $m$. Thus, by (2),

$$
\left(v^{\prime \prime}(x)\right)^{2} E\left(v^{\prime}\left(x+\xi_{m}\right)\right) E\left(v^{\prime \prime \prime}\left(x+\xi_{m}\right)\right)-v^{\prime}(x) v^{\prime \prime \prime}(x)\left(E\left(v^{\prime \prime}\left(x+\xi_{m}\right)\right)\right)^{2} \geq 0
$$

for every $m$. By (10), the left-hand side of (11) is equal to

$$
\begin{aligned}
& \left(v^{\prime \prime}(x)\right)^{2}\left(v^{\prime}(x)+\left(\frac{v^{\prime \prime \prime}(x)}{2}+\gamma_{m}^{1}\right) \sigma_{m}^{2}\right)\left(v^{\prime \prime \prime}(x)+\left(\frac{v^{(5)}(x)}{2}+\gamma_{m}^{3}\right) \sigma_{m}^{2}\right) \\
& -v^{\prime}(x) v^{\prime \prime \prime}(x)\left(v^{\prime \prime}(x)+\left(\frac{v^{(4)}(x)}{2}+\gamma_{m}^{2}\right) \sigma_{m}^{2}\right)^{2} .
\end{aligned}
$$

Rearranging these terms and dividing them by $\sigma_{m}^{2}$, we obtain

$$
\begin{aligned}
& \left(v^{\prime \prime}(x)\right)^{2} v^{\prime}(x)\left(\frac{v^{(5)}(x)}{2}+\gamma_{m}^{3}\right)+\left(v^{\prime \prime}(x)\right)^{2} v^{\prime \prime \prime}(x)\left(\frac{v^{\prime \prime \prime}(x)}{2}+\gamma_{m}^{1}\right) \\
- & v^{\prime}(x) v^{\prime \prime \prime}(x) 2 v^{\prime \prime}(x)\left(\frac{v^{(4)}(x)}{2}+\gamma_{m}^{2}\right) \\
+ & \left(\left(v^{\prime \prime}(x)\right)^{2}\left(\frac{v^{\prime \prime \prime}(x)}{2}+\gamma_{m}^{1}\right)\left(\frac{v^{(5)}(x)}{2}+\gamma_{m}^{3}\right)-\left(\frac{v^{(4)}(x)}{2}+\gamma_{m}^{2}\right)^{2}\right) \sigma_{m}^{2} \geq 0
\end{aligned}
$$

for every $m$. As $m \rightarrow \infty, \sigma_{m}^{2} \rightarrow 0$ and $\gamma_{m}^{n} \rightarrow 0$ for every $n$. Thus the left-hand side of (12) converges to

$$
\left(v^{\prime \prime}(x)\right)^{2} v^{\prime}(x) \frac{v^{(5)}(x)}{2}+\left(v^{\prime \prime}(x)\right)^{2} v^{\prime \prime \prime}(x) \frac{v^{\prime \prime \prime}(x)}{2}-v^{\prime}(x) v^{\prime \prime \prime}(x) 2 v^{\prime \prime}(x) \frac{v^{(4)}(x)}{2},
$$

and this is nonnegative, because it is the limit of a sequence of nonnegative numbers. By multiplying $2 / v^{\prime \prime}(x)$, which is negative, we obtain (5).

We shall now rewrite (5) in terms of $s(x)$ and its higher-order derivatives to arrive at (6). Since dividing expression $(5)$ by $\left(v^{\prime}(x)\right)^{3}$ does not change its sign,

$$
\frac{v^{\prime \prime}(x)}{v^{\prime}(x)}\left(\frac{v^{\prime \prime \prime}(x)}{v^{\prime \prime}(x)}\right)^{2}+\frac{v^{\prime \prime}(x)}{v^{\prime}(x)} \frac{v^{(5)}(x)}{v^{\prime}(x)}-2 \frac{v^{\prime \prime \prime}(x)}{v^{\prime}(x)} \frac{v^{(4)}(x)}{v^{\prime}(x)} \leq 0 .
$$

By definition,

$$
\frac{v^{\prime \prime}(x)}{v^{\prime}(x)}=-\frac{1}{s(x)} .
$$


Differentiating both sides of $-s(x) v^{\prime \prime}(x)=v^{\prime}(x)$ with respect to $x$ and using the above equality to eliminate $v^{\prime \prime}(x)$ from the expression, we obtain

$$
\frac{v^{\prime \prime \prime}(x)}{v^{\prime}(x)}=\frac{1+s^{\prime}(x)}{(s(x))^{2}}
$$

We can similarly obtain

$$
\begin{aligned}
& \frac{v^{(4)}(x)}{v^{\prime}(x)}=\frac{1}{(s(x))^{2}}\left(s^{\prime \prime}(x)-\frac{1}{s(x)}\left(1+s^{\prime}(x)\right)\left(1+2 s^{\prime}(x)\right)\right), \\
& \frac{v^{(5)}(x)}{v^{\prime}(x)}=\frac{1}{(s(x))^{2}}\left(s^{\prime \prime \prime}(x)-\frac{2}{s(x)}\left(2+3 s^{\prime}(x)\right) s^{\prime \prime}(x)+\frac{1}{(s(x))^{2}}\left(1+s^{\prime}(x)\right)\left(1+2 s^{\prime}(x)\right)\left(1+3 s^{\prime}(x)\right)\right) .
\end{aligned}
$$

Using these terms expression (14) is equal to

$$
\begin{aligned}
& \left(1+s^{\prime}(x)\right)^{2} \\
+ & \left((s(x))^{2} s^{\prime \prime \prime}(x)-2 s(x)\left(2+3 s^{\prime}(x)\right) s^{\prime \prime}(x)+\left(1+s^{\prime}(x)\right)\left(1+2 s^{\prime}(x)\right)\left(1+3 s^{\prime}(x)\right)\right) \\
+ & 2\left(1+s^{\prime}(x)\right)\left(s(x) s^{\prime \prime}(x)-\left(1+s^{\prime}(x)\right)\left(1+2 s^{\prime}(x)\right)\right)
\end{aligned}
$$

divided by $-(s(x))^{5}<0$. Then (5) is equivalent to this expression being nonnegative. Rearranging the terms, we obtain (6).

As for (7), we can analogously show that

$$
\begin{aligned}
\frac{v^{\prime \prime}(x)}{v^{\prime}(x)} & =-a(x), \\
\frac{v^{\prime \prime \prime}(x)}{v^{\prime}(x)} & =(a(x))^{2}-a^{\prime}(x), \\
\frac{v^{(4)}(x)}{v^{\prime}(x)} & =-(a(x))^{3}+3 a(x) a^{\prime}(x)-a^{\prime \prime}(x), \\
\frac{v^{(5)}(x)}{v^{\prime}(x)} & =(a(x))^{4}-6(a(x))^{2} a^{\prime}(x)+3\left(a^{\prime}(x)\right)^{2}+4 a(x) a^{\prime \prime}(x)-a^{\prime \prime \prime}(x) .
\end{aligned}
$$

Plugging these terms into expression (14) and rearranging the terms, we establish (7).

Suppose conversely that (5) holds as a strict inequality. Then (13) is strictly positive. Thus the right-hand side of (12) is strictly positive for every sufficiently large $m$. Since it is equal to the left-hand side of (11) divided by $\sigma_{m}^{2}>0$, this implies that the left-hand side of (11) is strictly positive. The proof is thus completed. 
The crucial step in the above proof was to ensure that the sequences $\left(\sigma_{m}^{2}\right)$ and $\left(\gamma_{m}^{n}\right)$, defined in (9), converge to zero as $m \rightarrow \infty$. This property, in turn, is guaranteed by (8), which asserts that all moments converge to zero $\left(E\left(\xi_{m}^{k}\right) \rightarrow 0\right)$ and also that the higher moments do so faster than the second moment $\left(E\left(\xi_{m}^{k}\right) / E\left(\xi_{m}^{2}\right) \rightarrow 0\right)$. The convergence of all moments does not always imply the faster convergence of higher moments. For example, if $\xi_{m}$ takes value 1 and -1 with probability $1 / m$ each, and 0 with probability $1-2 / m$, then, for every even $k, E\left(\xi_{m}^{k}\right)=2 / m \rightarrow 0$ and yet $E\left(\xi_{m}^{k}\right) / E\left(\xi_{m}^{2}\right)=1$ for every $m$. To eliminate such cases, we assumed in Theorem 1 that the sequence of supports converges to $\{0\}\left(b_{m} \rightarrow 0\right)$. Although the meaning of being small in "small risks" should be carefully specified, the need for convergence in supports has, as far as we know, not been explicitly stated in the relevant literature, such as Gollier and Pratt (1996).

To facilitate a comparison with Gollier and Pratt (1996)'s necessary and sufficient conditions for risk-vulnerability for small risks (inequality (12) in Gollier and Pratt (1996)), we define, for each $n \in\{1, \ldots, 5\}, a_{n}(x)=-v^{(n+1)}(x) / v^{(n)}(x)$ whenever $v^{(n)}(x) \neq 0$. Then $a_{1}(x)$ is nothing but the absolute risk aversion $a(x), a_{2}(x)$ is the absolute prudence $\psi(x)$, and $a_{3}(x)$ is what Kimball (1992) termed temperance. Then Gollier and Pratt (1996)'s necessary condition for risk vulnerability for small risks, derived from their expression (10), is that

$$
a_{2}(x)\left(a_{3}(x)-a_{1}(x)\right) \geq 0
$$

while the sufficient condition is obtained by replacing the weak inequality by a strict inequality in (15).

The following theorem restates the necessary and sufficient conditions of Theorem 1 in terms of the $a_{n}(x)$ when the decision maker is prudent.

Theorem 2 If $v^{\prime \prime \prime}(x)>0$, then (5) is equivalent to

$$
\frac{v^{\prime \prime \prime}(x)}{v^{\prime}(x)}+\frac{v^{(5)}(x)}{v^{\prime \prime \prime}(x)}-2 \frac{v^{(4)}(x)}{v^{\prime \prime}(x)} \geq 0 .
$$

If, in addition, $v^{(4)}(x) \neq 0$, then (16) is equivalent to each of the following three inequal- 
ities:

$$
\begin{aligned}
a_{3}(x)\left(a_{4}(x)-a_{2}(x)\right) & \geq a_{2}(x)\left(a_{3}(x)-a_{1}(x)\right), \\
2 a_{2}(x) a_{3}(x) & \leq a_{1}(x) a_{2}(x)+a_{3}(x) a_{4}(x), \\
\frac{d}{d x}\left(\frac{a_{2}(x)}{a_{1}(x)}\left(a_{3}(x)-a_{1}(x)\right)\right) & \leq 0 .
\end{aligned}
$$

The equivalence remains to hold when the weak inequalities in (16), (17), (18), and (19) are all replaced by strict inequalities.

Proof of Theorem 2 Inequality (16) follows from inequality (5) by dividing both sides by $v^{\prime}(x) v^{\prime \prime}(x) v^{\prime \prime \prime}(x)$, which is strictly negative. Further rearranging yields

$$
\frac{v^{(5)}(x)}{v^{\prime \prime \prime}(x)}-\frac{v^{(4)}(x)}{v^{\prime \prime}(x)} \geq \frac{v^{(4)}(x)}{v^{\prime \prime}(x)}-\frac{v^{\prime \prime \prime}(x)}{v^{\prime}(x)}
$$

and hence, if $v^{(4)}(x) \neq 0$, then

$$
-\frac{v^{(4)}(x)}{v^{\prime \prime \prime}(x)}\left(-\frac{v^{(5)}(x)}{v^{(4)}(x)}+\frac{v^{\prime \prime \prime}(x)}{v^{\prime \prime}(x)}\right) \geq-\frac{v^{\prime \prime \prime}(x)}{v^{\prime \prime}(x)}\left(-\frac{v^{(4)}(x)}{v^{\prime \prime \prime}(x)}+\frac{v^{\prime \prime}(x)}{v^{\prime}(x)}\right) .
$$

By the definition of the $a_{n}(x)$, the last inequality is equivalent to inequality (17), which after rearranging delivers inequality (18). (19) is equivalent to (18), because

$$
\frac{d}{d x}\left(\frac{a_{2}(x)}{a_{1}(x)}\left(a_{3}(x)-a_{1}(x)\right)\right)=\frac{a_{2}(x)}{a_{1}(x)}\left(2 a_{2}(x) a_{3}(x)-a_{1}(x) a_{2}(x)-a_{3}(x) a_{4}(x)\right) .
$$

Note that the right-hand side of (17) is exactly the same as the left-hand side of (15). Note moreover that the left-hand side of (17) is the same as its right-hand side except that every expression is of exactly one degree higher. This has a couple of easy-to-grasp implications. Suppose that $v$ is risk-vulnerable for small risks, i.e., the right-hand side of inequality (17) is positive. Then a necessary condition for $v$ to exhibit increased cautiousness under small risks is that the utility function $-v^{\prime}$ is also risk-vulnerable for small risks. For another implication, recall that inequality (10) in Gollier and Pratt (1996) shows that the percentage increase in absolute risk aversion due to a small zeromean background risk is proportional to $a_{2}(x)\left(a_{3}(x)-a_{1}(x)\right)$. Hence the expression 
$a_{2}(x)\left(a_{3}(x)-a_{1}(x)\right)$ can be interpreted as a measure of risk vulnerability for small risks. (17) says that $v$ exhibits increased cautiousness for small risks if and only if $-v^{\prime}$ is more risk-vulnerable than $v$. Finally, (19) states that the Gollier-Pratt measure of risk vulnerability divided by the Arrow-Pratt measure of absolute risk aversion is a decreasing function of consumption levels $x$ if and only if $v$ exhibits increasing cautiousness, and also if and only if $-v^{\prime}$ is more risk-vulnerable than $v$. This means that once we know the ratio between the Gollier-Pratt and Arrow-Pratt measures as a function of consumption levels, we can tell whether $v$ exhibits increased cautiousness for small risks.

\section{Sufficient condition for increased cautiousness}

In this section, we present a sufficient condition for the cautiousness to be increased by all zero-mean background risks, small or not.

Theorem 3 If $s^{\prime}(x) \geq 0, s^{\prime \prime}(x) \leq 0$, and $s^{\prime \prime \prime}(x) \geq 0$ for every $x>\underline{c}$, then for every zero-mean background risk $\zeta$ and for every $x>\underline{d}, t^{\prime}(x) \geq s^{\prime}(x)$. The inequality is strict if, in addition, $s^{\prime}(x) \neq 0$ for every $x>\underline{c}$.

This theorem says that at any given consumption level $x>\underline{d}$, the cautiousness $t^{\prime}(x)$ of the induced utility function $u$ is not exceeded by the cautiousness $s^{\prime}(x)$ of the original utility function $v$ if the cautiousness $s^{\prime}$ is a non-negative, non-increasing, and convex function of consumption levels. The first sign condition is nothing but non-increasing absolute risk aversion (DARA). The second sign condition is that the risk tolerance $s$ be concave, which implies that the absolute risk aversion $a$ is convex. ${ }^{5}$ It is easy to see that the three sign conditions imply the necessary and sufficient conditions (6 and 7) in Theorem 1 for the cautiousness to be increased by small risks, as well as the sufficient condition for risk vulnerability of (Gollier and Pratt, 1996, Corollary 1). Thus $t(x) \leq s(x)$. By Lemma $1, t^{\prime}(x) \geq s^{\prime}(x)$ if and only if $\varphi(x) t(x) \geq \psi(x) s(x)$. Hence $\varphi(x) \geq \psi(x)$, that is, the prudence is also increased by the background risk.

\footnotetext{
${ }^{5}$ The converse, however, does not hold. Even when the absolute risk aversion is convex, the absolute risk tolerance may not be concave. An undesirable implication of concave absolute risk tolerance, which is not implied by convex absolute risk aversion, is increasing relative risk aversion: Let $\underline{c}=0$, then, by the Inada condition, $s(x) \rightarrow 0$ as $x \rightarrow 0$. Thus the concavity of $s$ implies that its elasticity is not greater than one; and it is strictly less than one beyond any point at which $s^{\prime \prime}$ is strictly negative. But it can be shown that the elasticity is strictly less than one if and only if the first derivative of the relative risk aversion is strictly positive.
} 
Proof of Theorem 3 Let $x>\underline{d}$. By Lemma 1 and direct calculation,

$$
\begin{aligned}
t^{\prime}(x)= & \frac{E\left(v^{\prime}(x+\xi)\right) E\left(v^{\prime \prime \prime}(x+\xi)\right)}{\left(E\left(v^{\prime \prime}(x+\xi)\right)\right)^{2}}-1 \\
= & E\left(\frac{v^{\prime}(x+\xi) v^{\prime \prime \prime}(x+\xi)}{\left(v^{\prime \prime}(x+\xi)\right)^{2}} \frac{\left(v^{\prime \prime}(x+\xi)\right)^{2}}{v^{\prime}(x+\xi)}\right) \frac{E\left(v^{\prime}(x+\xi)\right)}{\left(E\left(v^{\prime \prime}(x+\xi)\right)\right)^{2}}-1 \\
= & E\left(\left(s^{\prime}(x+\xi)+1\right) \frac{\left(v^{\prime \prime}(x+\xi)\right)^{2}}{v^{\prime}(x+\xi)}\right) \\
& \times E\left(\frac{v^{\prime}(x+\xi)}{-v^{\prime \prime}(x+\xi)} \frac{-v^{\prime \prime}(x+\xi)}{E\left(-v^{\prime \prime}(x+\xi)\right)}\right) \frac{1}{E\left(-v^{\prime \prime}(x+\xi)\right)}-1 .
\end{aligned}
$$

By applying Jensen's inequality to the hyperbolic function and noting that the function $z \mapsto-v^{\prime \prime}(x+z) / E\left(-v^{\prime \prime}(x+\xi)\right)$ has the property of a Radon-Nikodym derivative, we obtain

$$
\begin{aligned}
& E\left(\frac{v^{\prime}(x+\xi)}{-v^{\prime \prime}(x+\xi)} \frac{-v^{\prime \prime}(x+\xi)}{E\left(-v^{\prime \prime}(x+\xi)\right)}\right) \\
\geq & \frac{1}{E\left(\frac{-v^{\prime \prime}(x+\xi)}{v^{\prime}(x+\xi)} \frac{-v^{\prime \prime}(x+\xi)}{E\left(-v^{\prime \prime}(x+\xi)\right)}\right)} \\
= & \frac{E\left(-v^{\prime \prime}(x+\xi)\right)}{E\left(\frac{\left(v^{\prime \prime}(x+\xi)\right)^{2}}{v^{\prime}(x+\xi)}\right)},
\end{aligned}
$$

where the weak inequality $\geq$ holds as a strict inequality unless $s^{\prime}(x+\xi)=0$. Thus

$$
t^{\prime}(x) \geq E\left(\left(s^{\prime}(x+\xi)+1\right) \frac{\left(v^{\prime \prime}(x+\xi)\right)^{2}}{v^{\prime}(x+\xi)}\right) \frac{1}{E\left(\frac{\left(v^{\prime \prime}(x+\xi)\right)^{2}}{v^{\prime}(x+\xi)}\right)}-1 .
$$

Since $-v^{\prime \prime}(x+z) / v^{\prime}(x+z)$ and $-v^{\prime \prime}(x+z)$ are non-increasing functions of $z$, so is their product $\left(v^{\prime \prime}(x+z)\right)^{2} / v^{\prime}(x+z)$. Since $s^{\prime \prime} \leq 0, s^{\prime}(x+z)$ is also a non-increasing function of $z$. Thus

$$
E\left(\left(s^{\prime}(x+\xi)+1\right) \frac{\left(v^{\prime \prime}(x+\xi)\right)^{2}}{v^{\prime}(x+\xi)}\right) \geq E\left(\left(s^{\prime}(x+\xi)+1\right)\right) E\left(\frac{\left(v^{\prime \prime}(x+\xi)\right)^{2}}{v^{\prime}(x+\xi)}\right)
$$


Hence

$$
t^{\prime}(x) \geq E\left(s^{\prime}(x+\xi)+1\right)-1=E\left(s^{\prime}(x+\xi)\right)
$$

Finally, since $s^{\prime \prime \prime}(x+z) \geq 0, s^{\prime}(x+z)$ is a convex function of $z$. Thus, by Jensen's inequality, $E\left(s^{\prime}(x+\xi)\right) \geq s^{\prime}(x)$, which completes the proof.

Although we assumed in this theorem that $s^{\prime}(x) \geq 0$ for every $x>\underline{c}$, this assumption can be derived from the assumption that $s^{\prime \prime}(x) \leq 0$ for every $x>\underline{c}$. Indeed, if $s^{\prime \prime}(x) \leq 0$ for every $x>\underline{c}$ and yet there were an $x_{0}>\underline{c}$ such that $s^{\prime}\left(x_{0}\right)<0$, then $s$ would be a concave and strictly decreasing function on $\left[x_{0}, \infty\right)$, and hence $s(x)<0$ for every sufficiently large $x>\underline{c}$, which is a contradiction. Thus, if $s^{\prime \prime}(x) \leq 0$ for every $x>\underline{c}$, then $s^{\prime}(x) \geq 0$ for every $x>\underline{c}$.

Although we imposed the three sign conditions over the entire domain $[\underline{c}, \infty)$ of consumption levels, the conclusion $t^{\prime}(x) \geq s^{\prime}(x)$ still holds as long as they are met on the interval $[x+\underline{e}, x+\bar{e}]$ of realizable consumption levels. Bear in mind, however, that $s^{\prime}$ need no longer be non-negative even if $s^{\prime \prime}$ is non-positive on $[x+\underline{e}, x+\bar{e}]$.

The conditions for Theorem 3 are satisfied by every HARA utility function, with the second and third derivatives being always zero.

Assumption 1 There exist a $\tau \in \boldsymbol{R}$ and a $\gamma \in \boldsymbol{R}_{++}$such that $s(x)=\tau+\gamma x$ for every $x>\underline{c}$.

Corollary 1 Under Assumption 1, if $\operatorname{Var}(\xi)>0$, then $t^{\prime}(x)>s^{\prime}(y)$ for every $x>\underline{d}$ and every $y>\underline{c}$

Corollary 1 shows that if the cautiousness $s^{\prime}$ of the original utility function $v$ is constant, then we have $t^{\prime}(x)>s^{\prime}(y)$ regardless of the choice of $x>\underline{d}$ and $y>\underline{c}$. This corollary could be used to provide an alternative and simple proof of Franke et al. (1998)'s Theorem 3.

\section{Consequences for portfolio selection}

In this section we consider how the cautiousness is related to portfolio choices in general, and the demand for call options in particular. The issue we deal with here is nothing intrinsic to background risks. Rather, we simply conduct a comparative statics exercise with respect to changes in cautiousness. If, for example, the sufficient condition of 
Theorem 3 is satisfied, then any zero-mean background risk would increase cautiousness, and we can then apply the results of this section to see how the portfolio demand would be affected by the presence of the background risk.

We first lay out the setting for the analysis of consumption choices. The risk the economy is faced with is described by a probability measure space $(\Omega, \mathscr{F}, P)$, for which the expectation operator is given by $E^{P}$, to distinguish it from the expectation operator $E=E^{Q}$ with respect to the background risk. The aggregate endowment of the economy is given by a measurable function $\zeta: \Omega \rightarrow\left(\underline{d}_{0}, \infty\right)$, where $\underline{d}_{0}$ is interpreted as the minimum subsistence level for the entire economy and is often taken to be zero, and the state-price density is $\pi:\left(\underline{d}_{0}, \infty\right) \rightarrow \boldsymbol{R}_{++}$.

The payoffs of all the assets we shall consider are functions of the aggregate endowment $\zeta$, and thus we identify an asset with a function $\varphi:\left(\underline{d}_{0}, \infty\right) \rightarrow \boldsymbol{R}$. The price at the time of transactions of an asset $\varphi$ is $E^{P}(\pi(\zeta) \varphi(\zeta))$.

A decision maker is characterized by his utility function $u:(\underline{d}, \infty) \rightarrow \boldsymbol{R}$ and his wealth level $w \in \boldsymbol{R}$. A consumption choice is characterized by a function $f:\left(\underline{d}_{0}, \infty\right) \rightarrow$ $(\underline{d}, \infty)$, so that his state-contingent consumption is $f(\zeta)$ and the corresponding expected utility level is $E^{P}(u(f(\zeta)))$. His utility maximization problem is therefore ${ }^{6}$

$$
\begin{array}{cc}
\max _{f} & E^{P}(u(f(\zeta))) \\
\text { subject to } & E^{P}(\pi(\zeta) f(\zeta)) \leq w .
\end{array}
$$

Let $t(x)=-u^{\prime}(x) / u^{\prime \prime}(x)$ and $t_{0}(x)=-\pi(x) / \pi^{\prime}(x)$, then $t$ is the absolute risk tolerance of the decision maker and $t_{0}$ can be considered as the absolute risk tolerance of the representative agent. Their derivatives, $t^{\prime}$ and $t_{0}^{\prime}$, are the cautiousness. It then follows from the first-order condition for utility maximization and part a) of Theorem 2 of Chevallier and Müller $(1994)^{7}$ that

$$
\frac{f^{\prime \prime}(x)}{f^{\prime}(x)}=\frac{1}{t_{0}(x)}\left(t^{\prime}(f(x))-t_{0}^{\prime}(x)\right)
$$

From this, we know that if a utility function always exhibits higher cautiousness than

\footnotetext{
${ }^{6}$ We are considering consumption plans that are contingent only on the aggregate endowment $\zeta$, but this involves no essential loss of generality. Since the state-price deflator $\pi$ is a function of $\zeta$ and the utility function $u$ exhibits risk aversion, he would optimally choose a single consumption level on any event over which the realized levels of $\zeta$ are common.

${ }^{7}$ The result was also given as Proposition 3 of Hara et al. (2007).
} 
another even when the consumption levels at which the two cautiousness are evaluated are not equal, then the curvature of the optimal consumption function $f$ is higher for the first utility function than for the second.

Proposition 2 Let $u_{1}:\left(\underline{d}_{1}, \infty\right) \rightarrow \boldsymbol{R}$ and $u_{2}:\left(\underline{d}_{2}, \infty\right) \rightarrow \boldsymbol{R}$ be utility functions satisfying the conditions at the beginning of Section 2. Assume that

$$
t_{1}^{\prime}\left(x_{1}\right) \geq t_{2}^{\prime}\left(x_{2}\right)
$$

for every $x_{1}>\underline{d}_{1}$ and every $x_{2}>\underline{d}_{2}$. Let $w_{1} \in \boldsymbol{R}$ and $w_{2} \in \boldsymbol{R}$ be wealth levels. Let $f_{1}:\left(\underline{d}_{0}, \infty\right) \rightarrow\left(\underline{d}_{1}, \infty\right)$ be the solution to the utility maximization problem (20) of $(u, w)=\left(u_{1}, w_{1}\right)$ and $f_{2}:\left(\underline{d}_{0}, \infty\right) \rightarrow\left(\underline{d}_{2}, \infty\right)$ be the solution to the utility maximization problem (20) of $(u, w)=\left(u_{2}, w_{2}\right)$. Then

$$
\frac{f_{1}^{\prime \prime}(x)}{f_{1}^{\prime}(x)} \geq \frac{f_{2}^{\prime \prime}(y)}{f_{2}^{\prime}(y)}
$$

for every $x>\underline{d}_{0}$ and every $y>\underline{d}_{0}$. The weak inequality holds as a strict inequality if the weak inequality of (22) holds as a strict inequality.

The utility maximization problem (20) is formulated in terms of a consumption function $f$, without specifying what kind of portfolio would implement the optimal consumption function. Let's now consider this implementation problem.

The types of assets of special interest are the risk-free bond, the market portfolio, and call options written on the market portfolio. They are characterized by the constant function taking values one, i.e., $\varphi(x)=1$ for every $x>\underline{d}$; the identity function, i.e., $\varphi(x)=x$ for every $x>\underline{d}$; and the piecewise-linear convex function $\varphi(x)=\max \{x-$ $k, 0\}=(x-k)^{+}$for every $x>\underline{d}$, where $k \in\left[\underline{d}_{0}, \infty\right)$ is the exercise price of the call option.

If the optimal consumption function $f:\left(\underline{d}_{0}, \infty\right) \rightarrow(\underline{d}, \infty)$ is affine, then it is enough to hold the risk-free bond and the market portfolio. Specifically, if there are a $\theta \in \boldsymbol{R}$ and a $\kappa \in \boldsymbol{R}$ such that $f(x)=\theta x+\kappa$ for every $x>\underline{d},{ }^{8}$ then the optimal portfolio consists of $\kappa$ units of the risk-free bond and $\theta$ units of the market portfolio. However, if $f$ is not affine, then it is necessary to hold options, possibly with infinitely many varieties of exercise prices, to implement $f$.

\footnotetext{
${ }^{8}$ Since both $u$ and $\pi$ satisfy the Inada condition, we in fact have $\kappa=\underline{d}-\theta \underline{d}_{0}$ and hence $f(x)=$ $\theta\left(x-\underline{d}_{0}\right)+\underline{d}$.
} 
We now formulate a portfolio of options of infinitely many exercise prices. First, denote by $\mathscr{G}$ the set of all right-continuous functions $G: \boldsymbol{R} \rightarrow \boldsymbol{R}$ such that its restriction on any interval of finite length is of bounded variation and that $G(x)=0$ for every $x<\underline{d}_{0} \cdot{ }^{9}$ For every $G \in \mathscr{G}$, there are two non-decreasing right-continuous functions, $G^{+}: \boldsymbol{R} \rightarrow \boldsymbol{R}_{+}$and $G^{-}: \boldsymbol{R} \rightarrow \boldsymbol{R}_{+}$, such that $G^{+}(x)=G^{-}(x)=0$ for every $x<\underline{d}_{0}$ and $G(x)=G^{+}(x)-G^{-}(x)$ for every $x \in \boldsymbol{R}$. Moreover, for every $x \in \boldsymbol{R}, G\left(\left[\underline{d}_{0}, x\right]\right)$ is bounded from both above and below, and hence the restriction of $G$ onto $\left[\underline{d}_{0}, x\right]$ induces a (unique) finite signed measure $M$ on the Borel $\sigma$-field $\mathscr{B}\left(\left[\underline{d}_{0}, x\right]\right)$ via $M((y, z])=G(y)-G(z)$ for every $y \in\left[\underline{d}_{0}, x\right]$ and $z \in\left[\underline{d}_{0}, x\right]$ with $y<z$. If either $G^{+}\left(\left[\underline{d}_{0}, \infty\right)\right)$ or $G^{-}\left(\left[\underline{d}_{0}, \infty\right)\right)$ is bounded from above, then $G$ induces a (unique) signed measure on $\mathscr{B}\left(\left[\underline{d}_{0}, \infty\right)\right)$ via $M((y, z])=G(y)-G(z)$.

A portfolio of assets can now be characterized as a triple $\left(y_{\mathrm{b}}, y_{\mathrm{m}}, G\right) \in \boldsymbol{R} \times \boldsymbol{R} \times \mathscr{G}$. Here $y_{\mathrm{b}}$ is interpreted as the units of the risk-free bond held in the portfolio; $y_{\mathrm{m}}$ as the units of the market portfolio; and $G$ is the distribution of the units of the call options held in the portfolio, identified by their exercise prices. The payoff given by portfolio $\left(y_{\mathrm{b}}, y_{\mathrm{m}}, G\right) \in \boldsymbol{R} \times \boldsymbol{R} \times \mathscr{G}$ is

$$
y_{\mathrm{b}}+y_{\mathrm{m}} x+\int_{\left[\underline{d}_{0}, \infty\right)}(x-k)^{+} d G(k)
$$

Strictly speaking, the integral of the last term need not be well defined because $G$ need not induce a signed measure. However, since $(x-k)^{+}=0$ for every $k \geq x$, we can think of this integral as being with respect to the finite signed measure induced by the restriction of $G$ onto $\left[\underline{d}_{0}, x\right]$. With this understanding, (23) is well defined. If, in particular, $G$ is continuous at $\underline{d}_{0}$ and differentiable on $\left(\underline{d}_{0}, \infty\right)$, with its derivative denoted by $g$, then (23) can be rewritten as

$$
y_{\mathrm{b}}+y_{\mathrm{m}} x+\int_{\underline{d}}^{x}(x-k) g(k) d k
$$

where the integral is a Riemann integral.

Let's now analyze how we can relate the optimal consumption function $f$ to the portfolio $\left(y_{\mathrm{b}}, y_{\mathrm{m}}, G\right)$. For this, note first that the Inada condition implies that $\lim _{x \downarrow \underline{d}_{0}} t(x)$

\footnotetext{
${ }^{9}$ Since the call option of exercise price $\underline{d}_{0}$ has the same payoff as the portfolio of holding one stock and selling $\underline{d}_{0}$ bonds, we could assume without loss of generality that $G\left(\underline{d}_{0}\right)=0$ as well. Then $G$ is continuous at $\underline{d}_{0}$.
} 
exists and is equal zero and $\lim _{x \downarrow \underline{d}_{0}} f(x)$ exists and is equal to $\underline{d}$. Moreover, $f$ is twice differentiable. We then obtain the following proposition regarding when and, if so, how to implement $f$ by holding a portfolio of the bond, the market portfolio, and call options.

Proposition 3 Let $f:(\underline{d}, \infty) \rightarrow \boldsymbol{R}$ be a twice differentiable consumption function. Then $f^{\prime}$ is of bounded variation on any sub-interval of $\left(\underline{d}_{0}, \infty\right)$ of finite length if and only if there exists a $\left(y_{\mathrm{b}}, y_{\mathrm{m}}, G\right) \in \boldsymbol{R} \times \boldsymbol{R} \times G$ such that

$$
f(x)=y_{\mathrm{b}}+y_{\mathrm{m}} x+\int_{\left[\underline{d}_{0}, \infty\right)}(x-k)^{+} d G(k)
$$

for every $x>\underline{d}_{0}$. Moreover, then, $\lim _{x \downarrow \underline{d}_{0}} f^{\prime}(x)$ exists and we can take $y_{\mathrm{b}}=\underline{d}-$ $\underline{d}_{0} \lim _{x \downarrow \underline{d}_{0}} f^{\prime}(x), y_{\mathrm{m}}=\lim _{x \downarrow \underline{d}_{0}} f^{\prime}(x)$, and

$$
G(k)=\left\{\begin{array}{cl}
0 & \text { if } k \leq \underline{d}_{0}, \\
f^{\prime}(k)-\lim _{x \downarrow \underline{d}_{0}} f^{\prime}(x) & \text { if } k>\underline{d}_{0} .
\end{array}\right.
$$

Since $G^{\prime}(k)=f^{\prime \prime}(k)$ by $(26)$, this proposition implies that $f^{\prime \prime}$ is the density function $G$. This fact has been noted by Leland (1980), (Huang, 2000, Proposition 1), (Huang, 2002, Proposition 2) and (Huang, 2005, Theorem 5). In these contributions, however, $f^{\prime}$ was not assumed to be of bounded variation. They obtained an equality of the sort

$$
f(x)=y_{\mathrm{b}}+y_{\mathrm{m}} x+\int_{\underline{d}_{0}}^{\infty}(x-k)^{+} f^{\prime \prime}(k) d k
$$

but this would not well defined if $f^{\prime}$ were not of bounded variation, because, then, the integrals of both positive and negative parts, $\int_{\underline{d}_{0}}^{\infty}(x-k)^{+}\left(f^{\prime \prime}(k)\right)^{+} d k$ and $\int_{\underline{d}_{0}}^{\infty}(x-k)^{+}\left(f^{\prime \prime}(k)\right)^{-} d k$, would be infinite.

Proof of Proposition 3 Suppose first that $f^{\prime}$ is of bounded variation. Then the image $f^{\prime}\left(\left(\underline{d}_{0}, x\right)\right)$ is bounded for each $x>\underline{d}_{0}$. Thus, if $\lim _{x \downarrow \underline{d}_{0}} f^{\prime}(x)$ did not exist, then there would exist two sequences of $\left(\underline{d}_{0}, \infty\right)$ that converges to $\underline{d}_{0}$ but the sequences of their values by $f^{\prime}$ have two different limits. This is a contradiction to the assumption that $f^{\prime}$ is of bounded variation. Thus $\lim _{x \downarrow \underline{d}_{0}} f^{\prime}(x)$ exists and we denote it by $\theta$. Define $\left(y_{\mathrm{b}}, y_{\mathrm{m}}, G\right)$ as in the proposition, then $G$ belongs to $\mathscr{G}$ and is differentiable satisfying 
$G^{\prime}=f^{\prime \prime}$ on $\left(\underline{d}_{0}, \infty\right)$. By integration by parts,

$$
\begin{aligned}
\int_{\left[\underline{d}_{0}, \infty\right)}(x-k)^{+} d G(k) & =\int_{\underline{d}_{0}}^{x}(x-k) f^{\prime \prime}(k) d k \\
& =\left[(x-k) f^{\prime}(k)\right]_{\underline{d}_{0}}^{x}+\int_{\underline{d}_{0}}^{x} f^{\prime}(k) d k \\
& =-\left(x-\underline{d}_{0}\right) \theta+(f(x)-\underline{d}) .
\end{aligned}
$$

Thus the right-hand side of (25) equals

$$
\underline{d}-\theta \underline{d}_{0}+\theta x-\left(x-\underline{d}_{0}\right) \theta+(f(x)-\underline{d})=f(x)
$$

We have thus established the existence of a $\left(y_{\mathrm{b}}, y_{\mathrm{m}}, G\right) \in \boldsymbol{R} \times \boldsymbol{R} \times G$ for which (25) holds.

Suppose conversely that there is a $\left(y_{\mathrm{b}}, y_{\mathrm{m}}, G\right) \in \boldsymbol{R} \times \boldsymbol{R} \times \mathscr{G}$ for which (25) holds. Define $h: \boldsymbol{R} \rightarrow \boldsymbol{R}$ by

$$
h(x)=\int_{\left[\underline{d}_{0}, \infty\right)}(x-k)^{+} d G(k) .
$$

Then $h$ is twice differentiable because $h(x)=f(x)-y_{\mathrm{b}}-y_{\mathrm{m}} x$. Moreover, for every $\varepsilon>0$,

$$
h(x+\varepsilon)-h(x)=\varepsilon G(x)+\int_{(x, x+\varepsilon]}(x+\varepsilon-k) d G(k) .
$$

Since $0 \leq x+\varepsilon-k \leq \varepsilon$ for every $k \in(x, x+\varepsilon]$,

$$
\begin{aligned}
& -\varepsilon\left(G^{-}(x+\varepsilon)-G^{-}(x)\right) \\
\leq & -\int_{(x, x+\varepsilon]}(x+\varepsilon-k) d G^{-}(k) \\
\leq & \int_{(x, x+\varepsilon]}(x+\varepsilon-k) d G(k) \\
\leq & \int_{(x, x+\varepsilon]}(x+\varepsilon-k) d G^{+}(k) \\
\leq & \varepsilon\left(G^{+}(x+\varepsilon)-G^{+}(x)\right) .
\end{aligned}
$$

Thus

$$
G(x)-\left(G^{-}(x+\varepsilon)-G^{-}(x)\right) \leq \frac{h(x+\varepsilon)-h(x)}{\varepsilon} \leq G(x)+\left(G^{+}(x+\varepsilon)-G^{+}(x)\right) .
$$


Since $G^{+}$and $G^{-}$are right-continuous, both the left- and right-hand sides of these inequalities converge to $G(x)$ as $\varepsilon \rightarrow \infty$. Thus $h^{\prime}(x)=G(x)$ for every $x>\underline{d}_{0}$. Since $h^{\prime}(x)=f^{\prime}(x)-y_{\mathrm{m}}$ for every $x>\underline{d}_{0}$ and $f^{\prime}$ is of bounded variation on any sub-interval of $\left(\underline{d}_{0}, \infty\right)$ of finite length, $G$ is also of bounded variation on any sub-interval of $\left(\underline{d}_{0}, \infty\right)$ of finite length.

If $f^{\prime}$ is of bounded variation and differentiable, we obtain the following result on the relationship between cautiousness and the optimal portfolio.

Proposition 4 Let $u_{1}, u_{2}, f_{1}$, and $f_{2}$ be as in Proposition 2. Let $\left(y_{\mathrm{b} 1}, y_{\mathrm{m} 1}, G_{1}\right)$ and $\left(y_{\mathrm{b} 2}, y_{\mathrm{m} 2}, G_{2}\right)$ be defined as in Proposition 3 for $f_{1}$ and $f_{2}$, respectively. Assume that $f_{1}^{\prime}$ and $f_{2}^{\prime}$ are differentiable and of bounded variation on any sub-interval of $\left(\underline{d}_{0}, \infty\right)$ of finite length. Then $G_{1}$ and $G_{2}$ are differentiable on $\left(\underline{d}_{0}, \infty\right)$ and

$$
\frac{G_{1}^{\prime}\left(k_{1}\right)}{y_{\mathrm{m} 1}+G_{1}\left(k_{1}\right)} \geq \frac{G_{2}^{\prime}\left(k_{2}\right)}{y_{\mathrm{m} 2}+G_{2}\left(k_{2}\right)}
$$

for every $k_{1}>\underline{d}_{0}$ and every $k_{2}>\underline{d}_{0}$. The weak inequality holds as a strict inequality if the weak inequality of (22) holds as a strict inequality.

This proposition compares the optimal portfolios of two decision makers with differing cautiousness. Since $G_{i}$ is the cumulative distribution function and $G_{i}^{\prime}$ is the density function of the portfolio of options, the fraction $G_{i}^{\prime}\left(k_{i}\right) /\left(y_{\mathrm{m} i}+G_{i}\left(k_{i}\right)\right)$ is the ratio of the units of options of exercise price $k_{i}$ held in the portfolio relative to the total units of the market portfolio and the options of exercise prices not higher than $k_{i}$. Thus the proposition states that the more cautious the decision maker is, the higher the percentage increase of option demands as a function of their exercise prices. Note that this does not mean that more options are held in the portfolio the more cautious the decision maker is. Rather, the result here is concerned with the units of options demanded relative to the units of options with lower exercise prices and the market portfolio. ${ }^{10}$

In some cases, $f^{\prime}$ is can be easily shown to be of bounded variation. For example, if the absolute risk tolerance of all decision makers are affine functions and their slopes are

\footnotetext{
${ }^{10}$ When the risk-free bond is available for trade, the market portfolio can be identified with the call option with exercise price $\underline{d}_{0}$. Then we can take $y_{\mathrm{m} i}=0$ while retaining the differentiability of $G_{i}$ on $\left(\underline{d}_{0}, \infty\right)$, and the fraction the fraction $G_{i}^{\prime}\left(k_{i}\right) /\left(y_{\mathrm{m} i}+G_{i}\left(k_{i}\right)\right)$ can be replaced by $G_{i}^{\prime}\left(k_{i}\right) / G_{i}\left(k_{i}\right)$ for every $k_{1}>\underline{d}_{0}$. Since $G_{i}^{\prime}\left(k_{i}\right) / G_{i}\left(k_{i}\right)=d\left(\ln G_{i}\left(k_{i}\right)\right) / d k_{i}$, Proposition 4 can then be restated as saying that the logarithm of the cumulative distribution function of option demands increases faster the more cautious the decision maker is.
} 
equal, then the efficient risk-sharing rules of all decision makers are affine functions, and thus of bounded variation. More generally, Propositions 4 and 8 of Hara (2007) imply that as long as the absolute risk tolerance of all decision makers are affine functions, possibly having different slopes, $f^{\prime}$ is of bounded variation.

There are, on the other hand, some cases in which $f^{\prime}$ is not of bounded variation.

Example 1 Let $\underline{d}=\underline{d}_{0}=0$. Define $h:[0,1] \rightarrow(1 / 3,2 / 3)$ by

$$
h(x)=\left\{\begin{array}{cl}
\frac{1}{2} & \text { if } x=0 \\
\frac{x}{6} \sin \left(\frac{1}{x}\right)+\frac{1}{2} & \text { otherwise. }
\end{array}\right.
$$

Then $h$ is continuous on $[0,1]$, continuously differentiable on $(0,1]$, but not of bounded variation. We extend $h$ to $\boldsymbol{R}_{+}$by setting $h(1)+h^{\prime}(1)(x-1)$ for every $x>1$. Then $h$ is continuous on $\boldsymbol{R}_{+}$and continuously differentiable on $\boldsymbol{R}_{++}$. We define $f_{1}: \boldsymbol{R}_{++} \rightarrow \boldsymbol{R}_{++}$ by $f_{1}(x)=\int_{0}^{x} h(y) d y$, then, $f_{1}$ is twice continuously differentiable, $f_{1}^{\prime}(x)=h(x) \in$ $(1 / 3,2 / 3)$ for every $x>0, f_{1}^{\prime}$ is not of bounded variation (although $\lim _{x \downarrow 0} f_{1}^{\prime}(x)$ exists and equals $1 / 2)$, and $f_{1}\left(\boldsymbol{R}_{++}\right)=\boldsymbol{R}_{++}$. Define $f_{2}: \boldsymbol{R}_{++} \rightarrow \boldsymbol{R}_{++}$by letting $f_{2}(x)=$ $x-f_{1}(x)$ for every $x>0$. Then $f_{1}(x)+f_{2}(x)=x$ and $f_{2}^{\prime}(x) \in(1 / 3,2 / 3)$ for every $x>0, f_{2}^{\prime}$ is not of bounded variation (although $\lim _{x \downarrow 0} f_{2}^{\prime}(x)$ exists and equals $1 / 2$ ), and $f_{2}\left(\boldsymbol{R}_{++}\right)=\boldsymbol{R}_{++}$. Let $\pi: \boldsymbol{R}_{++} \rightarrow \boldsymbol{R}_{++}$be any twice continuously differentiable function satisfying $\pi^{\prime}<0$ and the Inada condition. Then, by Theorems 2 and 3 of Hara (2007), for each $i$, there exists a three times continuously differentiable function $u_{i}: \boldsymbol{R}_{++} \rightarrow \boldsymbol{R}$ such that $u_{i}^{\prime \prime}<0<u_{i}^{\prime}$ and the Inada condition, and that $f_{1}$ and $f_{2}$ are optimal consumption functions for two decision makers having utility functions $u_{1}$ and $u_{2}$, and $\pi$ is the stateprice deflator supporting (decentralizing) $\left(f_{1}, f_{2}\right)$. Hence, if the economy is populated by these two decision makers and the aggregate wealth is appropriately distributed between the two, then each decision maker $i$ chooses the consumption $f_{i}(\zeta)$ such that $f_{i}^{\prime}$ is not of bounded variation.

\section{Conclusion}

The decision-maker's cautiousness (the derivative of the reciprocal of the Arrow-Pratt measure of absolute risk aversion) determines her demand for options relative to the risky asset. We have investigated how this cautiousness for macroeconomic risks is affected by 
the presence of idiosyncratic (background) risks and were interested in conditions under which cautiousness, and, hence, the demand for options relative to the risky asset, is increased in the presence of any background risk.

We gave a necessary and sufficient condition on a consumer's utility function to exhibit increased cautiousness under small background risks (Theorem 1), which, of course, is also a necessary condition for a consumer's utility function to exhibit increased cautiousness under any background risk. We also provided a sufficient condition (Theorem 3) on the original utility function under which the cautiousness, at any given level of consumption, is higher in the presence of a background risk than in its absence.

\section{References}

Brennan, M. J., Solanki, R., 1981. Optimal portfolio insurance. Journal of Financial and Quantitative Analysis 16 (3), 279-300.

Chevallier, E., Müller, H., 1994. Risk allocation in capital markets: Portfolio insurance, tactical asset allocation, and collar strategies. ASTIN Bulletin 24 (1), 5-18.

Franke, G., Stapleton, R. C., Subrahmanyam, M. G., 1998. Who buys and who sells options: The role of options in an economy with background risk. Journal of Economic Theory 82 (1), 89-109.

Gollier, C., 2001a. Economics of Time and Risk. MIT Press, Cambridge, Mass.

Gollier, C., Kimball, M. S., 1996. Towards a systematic approach to the economic effects of uncertainty: Characterizing utility functions, university of Michigan Discussion Paper.

Gollier, C., Pratt, J. W., 1996. Risk vulnerability and the tempering effect of background risk. Econometrica 64 (5), 1109-1123.

Hara, C., 2007. Necessary and sufficient conditions for the efficient risk-sharing rules and the representative consumer's utility function, working paper, Institute of Economic Research, Kyoto University.

Hara, C., Huang, J., Kuzmics, C., 2007. Representative consumer's risk aversion and efficient risk-sharing rules. Journal of Economic Theory 137 (1), 652-672. 
Huang, J., 2000. Who buys options from whom? The role of options in an economy with heterogeneous preferences and beliefs, lancaster University Management School Working Paper 2000/023 (revised on May 2002).

Huang, J., 2002. The role of options in an economy with background risk: A note, lancaster University Management School Working Paper 2002/013.

Huang, J., 2005. Background risk, cautiousness, and the roles of options: A note, lancaster University Management School, unpublished manuscript.

Huang, J., Stapleton, D., 2007. "Cautiousness" and measuring an investor's motive to buy options, unpublished manuscript.

Kihlstrom, R. E., Romer, D., Williams, S., 1981. Risk aversion with random initial wealth. Econometrica 49, 911-920.

Kimball, M. S., 1990. Precautionary saving in the small and in the large. Econometrica $58,53-73$.

Kimball, M. S., 1992. Precautionary motives for holding assets. In: Newman, P., Milgate, M., Eatwell, J. (Eds.), The New Palgrave Dictionary of Money and Finance. Stockton Press, New York, pp. 158-161.

Leland, H. E., 1980. Who should buy portfolio insurance. Journal of Finance 35 (2), 581-594.

Nachman, D. C., 1982. Preservation of "more risk averse" under expectations. Journal of Economic Theory 28, 361-368.

Wilson, R., 1968. The theory of syndicates. Econometrica 36 (1), 119-132. 